(2) Open Access Full Text Article

ORIGINAL RESEARCH

\title{
Association Between Different Obesity Patterns and the Risk of Developing Type 2 Diabetes Mellitus Among Adults in Eastern China: A Cross-Sectional Study
}

Yayun Lu'*

Hao Yang ${ }^{2, *}$

Zhiyue $\mathrm{Xu}^{\prime}$

Xuefeng Tang'

'Health Examination Center, Huadong Sanatorium, Wuxi, 214065, People's Republic of China; ${ }^{2}$ Department of Stomatology, Huadong Sanatorium, Wuxi, 214065, People's Republic of China

*These authors contributed equally to this work
Purpose: Obesity has become a public health challenge worldwide and can lead to the development of diabetes. However, studies examining the associations between different obesity patterns and the development of type 2 diabetes mellitus (T2DM) in China are limited. Therefore, this study aimed to explore the associations between three obesity patterns and the risk of T2DM development in Eastern China.

Methods: A cross-sectional study was conducted at our health examination center, involving 5860 adults, from June to December 2019. Data, including sociodemographic information, lifestyle, and biochemical measurements, were collected, and obesity was classified into three patterns: overweight and general obesity, abdominal obesity, and compound obesity. Multivariate logistic regression was used to assess the associations between different obesity patterns and T2DM risk after adjustment for confounding factors. Subgroup analysis was used to further explore the associations between obesity patterns and T2DM risk.

Results: A total of 5860 subjects were enrolled in this study. A significant difference in the T2DM incidence was observed between men and women with normal weight or overweight and general obesity $(p<0.05)$; however, no significant differences were observed between men and women with abdominal obesity and compound obesity. After multivariable adjustment, multivariate logistic regression analysis showed that the odds ratios (ORs) [95\% confidence interval (CI)] for T2DM in individuals with abdominal and compound obesity were 1.55 [1.08-2.24] and 1.85 [1.25-2.73], respectively, compared with the normal-weight group. Subgroup analysis showed that different obesity patterns were not independent risk factors for T2DM development among adults aged $\geq 60$ years, whereas abdominal and compound obesity were highly associated with the risk of T2DM development among individuals who report current smoking or alcohol drinking.

Conclusion: Abdominal obesity and compound obesity are risk factors for T2DM. More attention should be paid to obesity prevention among individuals younger than 60 years and improving control of cigarette and alcohol abuse.

Keywords: association, general obesity, abdominal obesity, type 2 diabetes

\section{Introduction}

Diabetes mellitus represents a huge public health burden worldwide, and 440 million people have been diagnosed with diabetes in global. The prevalence of type 2 diabetes mellitus (T2DM) has increased dramatically over recent decades in the Asia-Pacific 
region. ${ }^{1,2}$ China, with a population of 1.38 billion people, also features the largest population of individuals with diabetes (110 million), which has resulted in a great burden on the national healthcare institutions. ${ }^{3}$ T2DM is a multifactorial disease associated with various genetic and environmental factors, including obesity, aging, high-energy and high-fat dietary intake, and sedentary lifestyles, which have contributed to the high prevalence of diabetes in China during recent years. 4

Obesity is often associated with numerous complications, including cardiovascular disease and metabolic disorders, such as T2DM, and obesity and diabetes share a common insulin resistance pathway and contribute to chronic hyperglycemia. ${ }^{5,6}$ The associations between obesity and T2DM have long been recognized by past studies. The characteristics of fat distribution can be used to classify obesity into either general obesity or abdominal obesity. Body mass index (BMI) often serves as a common indicator for identifying general obesity, ${ }^{7}$ and waist circumference (WC) can be used to determine the status of abdominal fat accumulation and is often used to define abdominal obesity. ${ }^{8-10}$ A recent cohort study investigated a population of adolescents to explore the association between obesity and the risk of developing T2DM in adulthood, and the results showed that increased BMI during adolescence was related to a high risk of developing T2DM in adulthood. ${ }^{11}$ Another study also demonstrated that abdominal obesity was highly associated with an increased risk of T2DM. ${ }^{12}$ Therefore, different obesity patterns might represent significant risk factors for T2DM development.

However, few studies have explored the different levels of T2DM risk associated with different obesity patterns. In addition, individuals with general obesity may also present with abdominal obesity, which is referred to as compound obesity. Previous studies may have classified individuals with compound obesity in either general obesity or abdominal obesity groups, and the combination of general obesity and abdominal obesity may have additive effects on the risk of developing T2DM. Therefore, previously determined risks of T2DM associated with general or abdominal obesity may not be accurate due to the misclassification of compound obesity.

To address these issues, a cross-sectional study was conducted to explore the association between different obesity patterns and the risk of developing T2DM. We also performed subgroup analyses to examine the associations of different obesity patterns and the risk of T2DM compared with normal-weight individuals.

\section{Materials and Methods}

\section{Study Design and Population}

This cross-sectional study was initiated at our health examination center from June to December 2019. During this period, All participants attended the health examination were investigated, and 6,530 subjects were initially enrolled.The exclusion criteria were as follows: (1) incomplete clinical data; (2) receiving the treatment for diabetes in the past three months; (3) severe mental illness; (4) severe cardiovascular and cerebrovascular diseases, such as coronary heart disease, arrhythmia, or stroke; (5) severe liver or kidney dysfunction; (6) malignant tumors and autoimmune diseases; (7) the inability to complete the investigation independently. Finally, 5,860 subjects older than 18 years were enrolled in this study based on multistage random cluster sampling. This study was approved by the Ethics Committee of the Huadong Sanatorium (NO.ECHS2021-03).

Participants completed a questionnaire administered by a well-trained interviewer; demographic characteristics included sex and age in years $(<60$ years or $\geq 60$ years); lifestyle factors included the smoking and alcohol intake status, with smoking defined as more than 3 cigarettes per day for 12 sequential months, and drinking defined as alcohol consumption at least 3 times per week for at least 12 months; and personal medical history, including the diagnosis of chronic diseases, such as hypertension, diabetes, or cardiovascular disease.

\section{Assessment of T2DM and Hypertension} T2DM was defined as fasting blood glucose $(\mathrm{FBG}) \geq 7.0$ $\mathrm{mmol} / \mathrm{L}$ or participants' self-reporting of continuous insulin and antidiabetic treatments. ${ }^{13}$ Systolic and diastolic blood pressure was measured by an electronic brachial sphygmomanometer (T30J, OMRON, Japan). While in a seated position, participants were measured three times on the upper arm at 5-min intervals. Participants were also required not to partake in smoking, alcohol drinking, caffeine, tea, or excessive exercise 30 minutes before blood pressure measurements and were asked to remain silent during measurements. Hypertension was defined as systolic blood pressure (SBP) $\geq 140 \mathrm{mmHg}$ or diastolic blood pressure $(\mathrm{DBP}) \geq 90 \mathrm{mmHg}$, or currently using any antihypertensive medications. ${ }^{14}$

\section{Anthropometric Measurements}

Anthropometric variables included height, weight, WC, and hip circumference (HC). All participants were 
measured in light clothing and bare feet. Weight and height were measured twice and rounded to the nearest $0.1 \mathrm{~kg}$ and $0.5 \mathrm{~cm}$, respectively. BMI, as an indicator of general obesity, was calculated as body weight (in $\mathrm{kg}$ )/ [height (in $\mathrm{m})]^{2}$. WC, as an indicator of abdominal obesity, was measured between the costal margin and the iliac crest to the nearest $0.5 \mathrm{~cm}$ while participants gently exhaled. ${ }^{15}$

\section{Definition of Different Obesity Patterns}

BMI ranging from 18.5 to $23.9 \mathrm{~kg} / \mathrm{m}^{2}$ and was regarded as normal weight; overweight was defined as BMI $\geq 24.0 \mathrm{~kg} /$ $\mathrm{m}^{2}$, and general obesity was defined as BMI $\geq 28.0 \mathrm{~kg} / \mathrm{m}^{2}$ without an abnormal WC, based on the Chinese standard. $^{16}$ According to the guidelines of the International Diabetes Federation (IDF) for the Chinese population, abdominal obesity was defined as $\mathrm{WC} \geq$ $90 \mathrm{~cm}$ for men and $\mathrm{WC} \geq 80 \mathrm{~cm}$ for women, with BMI in the normal range. ${ }^{17}$ Compound obesity was defined as the coexistence of both general and abdominal obesity.

\section{Clinical Measurements}

Blood samples were collected for laboratory assays after at least 8 hours of an overnight fast, and blood samples were used to examine the levels of high-density lipoprotein cholesterol (HDL-c), low-density lipoprotein cholesterol (LDL-c), triglycerides (TG), total cholesterol (TC), FBG, glycated hemoglobin (HbA1c), white blood cells (WBC), neutrophils (NE) and lymphocytes (LY). The neutrophil to lymphocyte ratio (NLR) was calculated as the ratio of neutrophils to lymphocytes.

\section{Statistical Analysis}

Continuous variables are presented as the mean \pm standard deviation (SD), or the median (interquartile range), depending on whether the variables are normally distributed, as tested using the Shapiro-Wilk test. Categorical variables are presented as the number (percentage). We compared participants' characteristics using a Chi-square test for categorical variables and an analysis of variance (ANOVA) or Kruskal-Wallis test for continuous variables. To assess the associations between different obesity patterns and the risk of T2DM, multiple logistic regression analysis was conducted after controlling for confounding factors. Normal-weight individuals were used as the reference group, and the analysis was adjusted for potential confounders, including age, sex, smoking, drinking, hypertension, TG, HDL-C, and WBC. Subgroup analysis was also performed, stratified by age $(<60$ or $\geq 60$ years), sex (male or female), smoking (yes or no), and drinking (yes or no). Because overweight is also a risk factor for diabetes and the percentage of the population classified as general obesity was limited, we merged the overweight and general obesity populations into a single group. A two-tailed $p$-value $<0.05$ was considered significant. All statistical analyses were performed using SPSS 25.0 software package (SPSS Institute, Chicago).

\section{Results}

\section{Characteristics of the Study Population}

A total of 5,860 participants $(3,435$ men and 2,425 women) was enrolled in our study (Table 1). The prevalences of overweight and general obesity, abdominal obesity, and compound obesity were 1,421 (24.25\%), 974 (16.62\%), and 649 (11.08\%), respectively. The incidences of T2DM among the overweight and general obesity, abdominal obesity, and compound obesity groups were $77(5.4 \%), 81(8.3 \%)$, and $66(10.2 \%)$, respectively. A significant difference in the incidence of T2DM was observed among men and women between the normalweight and overweight and general obesity groups $(p<$ $0.05)$, whereas no significant difference in T2DM was observed among men and women between the abdominal obesity and compound obesity groups (Figure 1). Significant differences in demographic characteristics, lifestyle factors, and blood parameters were observed among the different obesity patterns (all $p<0.001$ ).

\section{Association Between Different Obesity Patterns and the Risk of T2DM}

All of the assessed obesity patterns were significantly associated with T2DM risk in the unadjusted model (Table 2). However, the associations between the three obesity patterns and the risk of T2DM substantially decreased after adjusting for confounding factors. Overweight and general obesity were not identified as independent factors associated with T2DM incidence in Model 2. In the fully adjusted Model 2, individuals with abdominal obesity had a 1.55 -fold increase in the odds of developing T2DM (95\% CI: 1.08-2.24; $p=0.018$ ) compared with individuals with normal weight at baseline. People with compound obesity were nearly twice as likely to develop T2DM (adjusted OR:1.85, 95\% CI: 1.25-2.73; $p=0.002)$. 
Table I Comparison of Patients' Characteristics Among Different Obesity Patterns $(n=5860)$

\begin{tabular}{|c|c|c|c|c|c|}
\hline Variables & $\begin{array}{l}\text { Normal Weight } \\
\qquad(n=28 \mid 6)\end{array}$ & $\begin{array}{l}\text { Overweight and General Obesity } \\
\qquad(n=\mid 42 I)\end{array}$ & $\begin{array}{l}\text { Abdominal Obesity } \\
\qquad(n=974)\end{array}$ & $\begin{array}{l}\text { Compound Obesity } \\
\qquad(n=649)\end{array}$ & $p$-value \\
\hline $\begin{array}{l}\text { Age }(\mathrm{n}, \%) \\
\quad \geq 60 \text { years } \\
<60 \text { years }\end{array}$ & $\begin{array}{l}312(11.1 \%) \\
2504(88.9 \%)\end{array}$ & $\begin{array}{l}176(12.4 \%) \\
1245(87.6 \%)\end{array}$ & $\begin{array}{l}225(23.1 \%) \\
75 ।(76.9 \%)\end{array}$ & $\begin{array}{l}94(14.5 \%) \\
555(85.5 \%)\end{array}$ & $<0.001$ \\
\hline Male $(n, \%)$ & 1109 (39.4\%) & $1002(70.5 \%)$ & 789 (80.8\%) & $536(82.6 \%)$ & $<0.001$ \\
\hline BMI $\left(\mathrm{kg} / \mathrm{m}^{2}\right)$ & $21.91[20.45,22.98]$ & $25.35[24.61,26.31]$ & $26.20[25.25,27.05]$ & $29.55[28.63,30.86]$ & $<0.001$ \\
\hline$W C(\mathrm{~cm})$ & $75.50 \pm 6.63$ & $84.13 \pm 4.12$ & $91.87 \pm 3.38$ & $97.53 \pm 6.48$ & $<0.001$ \\
\hline $\mathrm{HC}(\mathrm{cm})$ & $91.25 \pm 13.69$ & $96.00 \pm 3.39$ & $98.16 \pm 3.56$ & $103.64 \pm 5.03$ & $<0.001$ \\
\hline Smoking $(n, \%)$ & $423(15.0 \%)$ & $377(26.5 \%)$ & $380(38.9 \%)$ & $246(37.9 \%)$ & $<0.001$ \\
\hline Drinking $(\mathrm{n}, \%)$ & $271(9.6 \%)$ & $265(18.6 \%)$ & $250(25.6 \%)$ & $164(25.3 \%)$ & $<0.001$ \\
\hline SBP $(\mathrm{mmHg})$ & $113.73 \pm 14.62$ & $122.81 \pm 15.19$ & $124.60 \pm 14.55$ & $131.14 \pm 15.73$ & $<0.001$ \\
\hline $\mathrm{DBP}(\mathrm{mmHg})$ & $68.98 \pm 9.41$ & $74.34 \pm 10.10$ & $74.84 \pm 10.37$ & $80.56 \pm 11.06$ & $<0.001$ \\
\hline Hypertension (n,\%) & $137(4.9 \%)$ & $173(12.3 \%)$ & 137 (14.3\%) & $|6|(25.0 \%)$ & $<0.001$ \\
\hline $\mathrm{TG}(\mathrm{mmol} / \mathrm{L})$ & $0.94[0.67,1.40]$ & $1.42[0.98,2.14]$ & $1.65[1.16,2.40]$ & $1.84[1.32,2.66]$ & $<0.001$ \\
\hline $\mathrm{TC}(\mathrm{mmol} / \mathrm{L})$ & $4.66[4.11,5.24]$ & $4.79[4.23,5.39]$ & $4.76[4.27,5.35]$ & $4.79[4.32,5.43]$ & $<0.001$ \\
\hline LDL-C (mmol/L) & $2.86[2.45,3.32]$ & $3.06[2.63,3.50]$ & $3.07[2.64,3.47]$ & $3.08[2.71,3.52]$ & $<0.001$ \\
\hline HDL-C (mmol/L) & $1.44[1.23,1.66]$ & $1.23[1.07,1.40]$ & $1.17[1.03,1.35]$ & $1.13[0.99,1.28]$ & $<0.001$ \\
\hline $\mathrm{FBG}(\mathrm{mmol} / \mathrm{L})$ & $4.87[4.63,5.16]$ & $5.07[4.78,5.48]$ & $5.20[4.90,5.74]$ & $5.29[4.92,5.91]$ & $<0.001$ \\
\hline Diabetes (n,\%) & 71 (2.5\%) & 77 (5.4\%) & 81 (8.3\%) & $66(10.2 \%)$ & $<0.001$ \\
\hline HbAlc (\%) & $5.50[5.30,5.70]$ & $5.60[5.40,5.80]$ & $5.70[5.50,5.90]$ & $5.80[5.50,6.10]$ & $<0.001$ \\
\hline WBC $\left(\times 10^{9} / \mathrm{L}\right)$ & $5.47[4.70,6.40]$ & $5.80[4.95,6.90]$ & $6.00[5.10,7.00]$ & $6.25[5.32,7.20]$ & $<0.001$ \\
\hline $\mathrm{NE}\left(\times 10^{9} / \mathrm{L}\right)$ & $2.97[2.43,3.66]$ & $3.14[2.58,3.88]$ & $3.21[2.69,3.94]$ & $3.36[2.80,4.13]$ & $<0.001$ \\
\hline $\operatorname{LY}\left(\times 10^{9} / L\right)$ & $1.84[1.42,2.23]$ & $1.97[1.53,2.40]$ & $1.93[1.35,2.41]$ & $2.11[1.63,2.49]$ & $<0.001$ \\
\hline NLR & $1.60[1.26,2.36]$ & $1.61[1.26,2.25]$ & $\mathrm{I} .70[1.3 \mathrm{I}, 2.47]$ & $1.76[1.31,2.27]$ & $<0.001$ \\
\hline
\end{tabular}

Notes: Normally distributed continuous variables are presented as the mean \pm standard deviation; Non-normally distributed continuous variables are presented as the mean [interquartile range]; Categorical variables are presented as the number (percentage).

Abbreviations: BMI, body mass index; WC, waist circumference; HC, hip circumference; SBP, systolic blood pressure; DBP, diastolic blood pressure; TG, triglycerides; TC, total cholesterol; LDL-C, low-density lipoprotein cholesterol; HDL-c, high-density lipoprotein cholesterol; FBG, fasting blood glucose; HbAIc, glycated hemoglobin; WBC, white blood cells; NE, neutrophils; LY, lymphocytes; NLR, neutrophil to lymphocytes ratio.

\section{Subgroup Analysis of Different Obesity Patterns and the Risk of Developing T2DM} After subgroup analyses stratified by age, sex, smoking, and drinking, the risk of T2DM was found to increase for all three obesity patterns compared with normal weight (Figures 2-4), and all subgroup analyses were conducted after adjusting for age, sex, smoking, and drinking. People with compound obesity continued to be associated with a high-risk value of developing T2DM (Figure 3).
However, obesity patterns were not associated with diabetes risk in the population older than 60 years. Neither overweight and general obesity nor abdominal obesity were correlated with T2DM in women (all $p>0.05$; Figures 2 and 3); however, among women with compound obesity, the correlation was significant $(p<0.05)$. Abdominal obesity and compound obesity were identified as independent risk factors of T2DM development among smokers and alcohol drinkers $(p<0.05)$. 


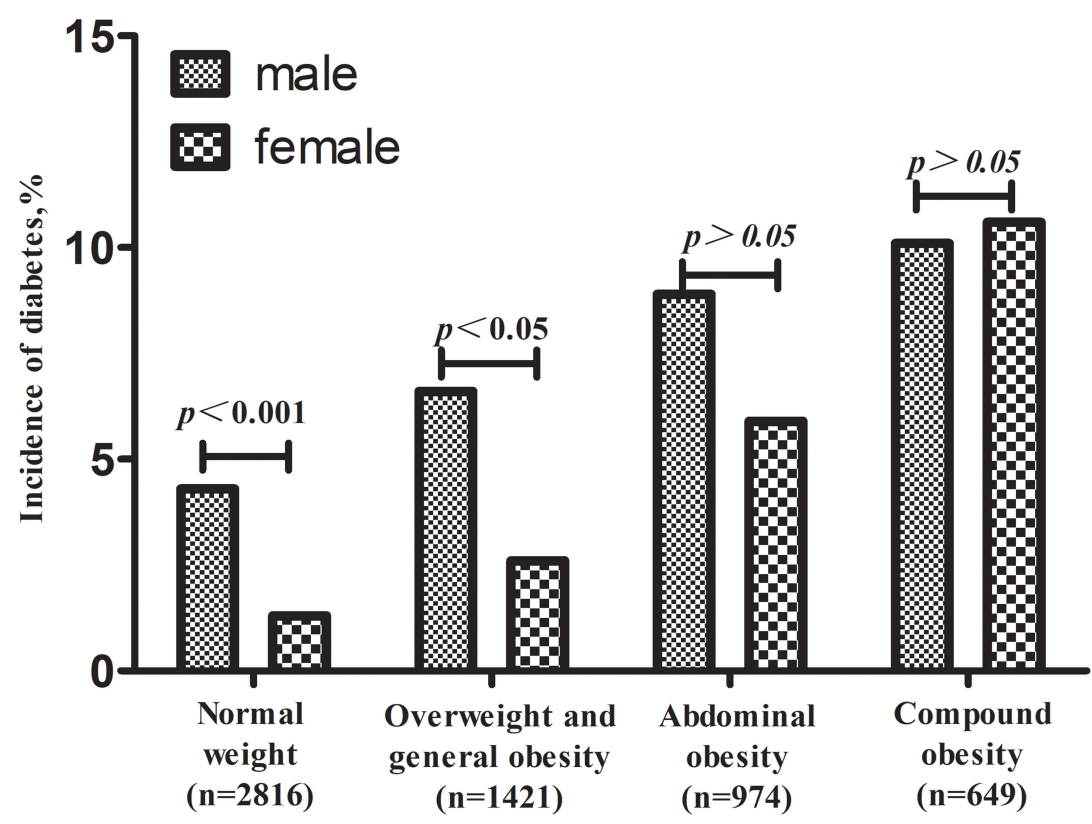

Figure I Comparison of the T2DM incidence among different obesity patterns $(n=5860)$.

\section{Discussion}

This cross-sectional study enrolled 5,860 participants and investigated the associations between different obesity patterns and the risk of developing T2DM. We used BMI as an indicator for general obesity and $\mathrm{WC}$ as an index for abdominal obesity. The incidences of diabetes in compound obesity, abdominal obesity, and overweight and general obesity were $10.2 \%, 8.3 \%$, and $5.4 \%$, respectively. Moreover, multivariable regression analysis showed that abdominal obesity and compound obesity were significantly associated with T2DM after adjusting for confounding factors. Different obesity patterns were not found to be independent risk factors for developing T2DM among individuals older than 60 years. In addition, abdominal and compound obesity were highly associated with the risk of developing T2DM among people who partake in smoking or alcohol drinking.

People with overweight and general obesity without abdominal obesity showed no significant association with T2DM risk after adjusting for confounding factors. Most studies have used BMI as the primary criteria for the assessment of general obesity; however, BMI does not accurately capture information regarding the adipose distribution throughout the body and is unable to differentiate fat mass from muscle mass. The observed influence on the risk of cardiometabolic symptoms was higher for body fat distribution than for BMI. Thus, visceral adipose tissues appear to have a great impact on the development of obesity-related metabolic disorders. ${ }^{18,19} \mathrm{WC}$, which is an index of body fat distribution, could serve as

Table 2 Multivariate Logistic Regression Model of Associations Between Different Patterns of Obesity and T2DM Risk ( $\mathrm{n}=5860)$

\begin{tabular}{|l|c|c|c|c|c|c|c|c|}
\hline Obesity Patterns & \multirow{2}{*}{ Total } & Diabetes & \multicolumn{2}{|c|}{ Unadjusted Model } & \multicolumn{2}{|c|}{ Model I } & \multicolumn{2}{c|}{ Model 2} \\
\cline { 5 - 9 } & & $(\mathbf{n}, \%)$ & OR [95\% Cl] & p-value & OR [95\% CI] & p-value & OR [95\% CI] & p-value \\
\hline Normal weight & 2816 & $71(2.5 \%)$ & 1.00 & - & 1.00 & - & 1.00 \\
\hline $\begin{array}{l}\text { Overweight and general } \\
\text { obesity }\end{array}$ & 1421 & $77(5.4 \%)$ & $2.21[1.59,3.08]$ & $<0.001$ & $1.78[1.26,2.49]$ & 0.001 & $1.38[0.97,1.96]$ & 0.075 \\
\hline Abdominal obesity & 974 & $81(8.3 \%)$ & $3.50[2.52,4.86]$ & $<0.001$ & $2.46[1.74,3.48]$ & $<0.001$ & $1.55[1.08,2.24]$ & 0.018 \\
\hline Compound obesity & 649 & $66(10.2 \%)$ & $4.38[3.10,6.19]$ & $<0.001$ & $3.09[2.15,4.45]$ & $<0.001$ & $1.85[1.25,2.73]$ & 0.002 \\
\hline
\end{tabular}

Notes: Model I, adjusted for age, sex, smoking, and drinking; Model 2, adjusted for age, sex, smoking, drinking, hypertension, TG, HDL-C, and WBC. 


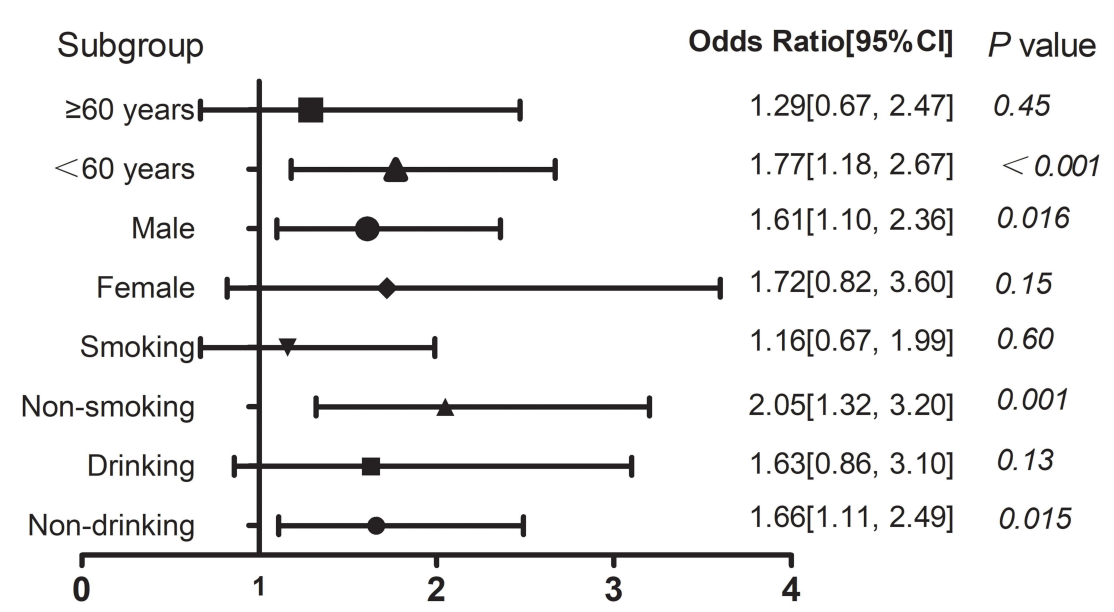

Figure 2 Subgroup analyses for the risks of developing T2DM in the overweight and general obesity group compared with the normal-weight group.

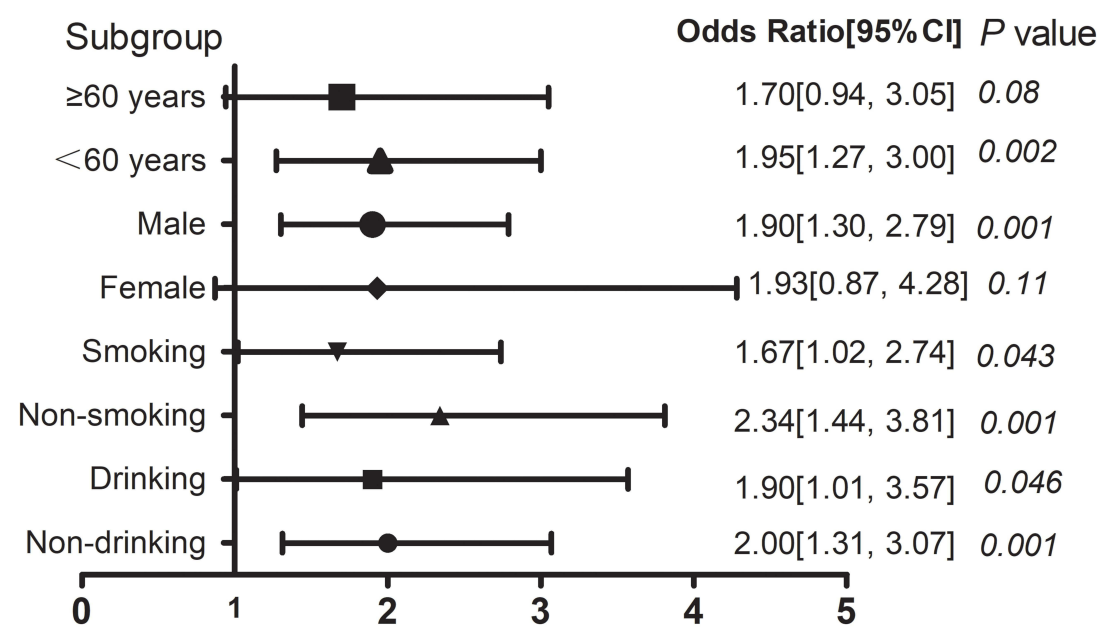

Figure 3 Subgroup analyses for the risks of developing T2DM in the group with abdominal obesity compared with the normal-weight group.

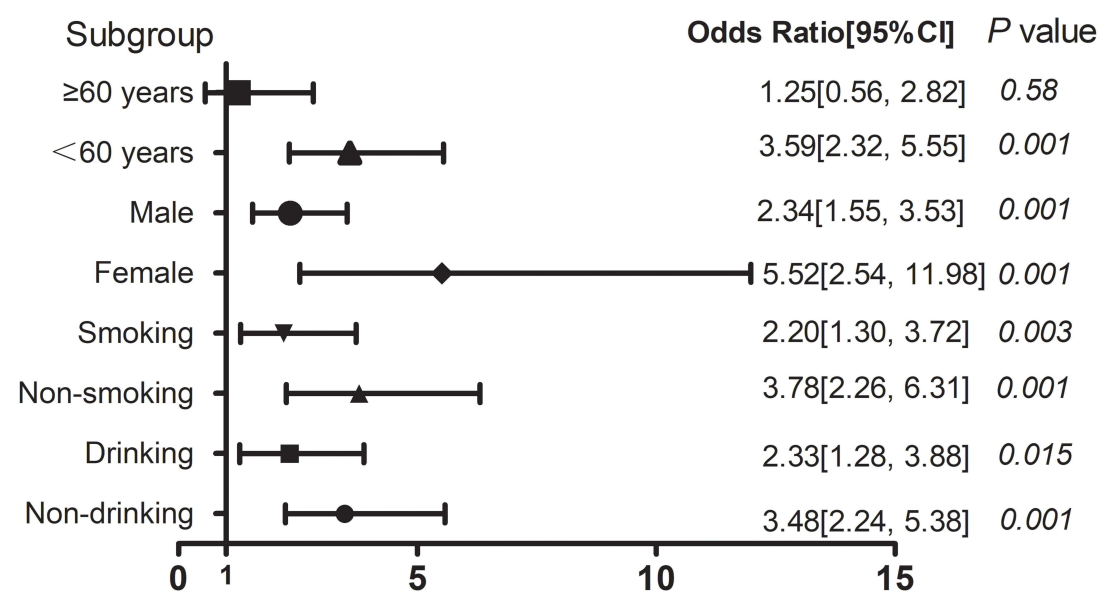

Figure 4 Subgroup analyses for the risks of developing T2DM in the group with compound obesity compared with the normal-weight group. 
a standardized parameter for assessing abdominal obesity, and the distribution of adipose tissue is a well-documented and significant factor associated with the development of insulin resistance. ${ }^{20}$ People with abdominal obesity but without general obesity showed a high risk of developing diabetes. The combination of both general and abdominal obesity displayed an additive effect on diabetes risk. The subgroup analysis indicated that women with compound obesity were more likely to develop diabetes compared with women of normal weight.

Subgroup analysis also indicated that general obesity and abdominal obesity were not independent risk factors of T2DM among people older than 60 years. Because hypofunction and metabolic disturbance can easily develop among older individuals, the effects of obesity on diabetes development were not obvious, and diabetes development is likely to be affected by chronic inflammation or sarcopenia. ${ }^{21}$ Older individuals commonly experience a loss of muscle mass and are more susceptible to the development of insulin resistance. ${ }^{22}$ Therefore, physical exercise is recommended as a sufficient method for improving muscle strength and mass. Abdominal obesity was identified as an independent risk factor for T2DM among people who partake in smoking and drinking. A recent study indicated that childhood exposure to parental smoking was associated with an increased risk of overweight and abdominal obesity. ${ }^{23}$ Another study showed that obesity was highly related to tobacco smoking. ${ }^{24}$ Active and passive smoking are typically accompanied by increased weight, and smokers tend to be either obese or overweight. Furthermore, in our study, people with abdominal obesity were more likely to partake in smoking (38.9\%); therefore, smoking may influence the development of metabolic disorders and induce the abnormal distribution of fat. In addition, alcohol drinking is known to be associated with the risk of hepatic metabolic functional damage, which could further affect glucose and lipid metabolism. In this study, a high percentage of people with abdominal obesity were also alcohol drinkers, and drinking alcohol drinking might lead to visceral fat accumulation. Moreover, women with general obesity or abdominal obesity were not as susceptible to T2DM development compared with men; however, females with compound obesity deserve more attention because the coexistence of general obesity and abdominal obesity has an additive effect on the risk of developing T2DM.
Abdominal obesity represents an abnormal adipose distribution phenotype and has been associated with insulin resistance and chronic inflammation. ${ }^{25}$ Macrophage infiltration has been identified in abdominal adipose tissue, and pro-inflammatory cytokines produced by macrophages have been shown to affect insulindependent tissues and beta cells. In our study, people with abdominal obesity displayed high NLR values compared with people with normal weight (1.70 [1.31, 2.47] vs $1.60[1.26,2.36])$, indicating that inflammation is correlated with abdominal obesity. Recent studies have also demonstrated that abdominal obesity was a strong predictor for incident diabetes. ${ }^{26,27}$ Another study illustrated that obesity and T2DM are associated with increased levels of oxidative stress, ${ }^{28}$ and several oxidative stress markers have been associated with diabetes. $^{29}$ Adipose tissue represents the largest endocrine organ and produces adipokines that stimulate the generation of oxidative substances, which can further affect the occurrence of diabetes. Therefore, programs and strategies for controlling obesity should be established and strengthened to reduce the incidence of T2DM in China. Several studies have explored effective interventions for preventing and controlling obesity, and a recent meta-analysis showed that probiotic and synbiotic supplementation could decrease the levels of oxidative stress among adults. Another study demonstrated that fasting and energy-restricted diets could reduce serum leptin concentrations. ${ }^{30}$ Meanwhile, a recent systematic review and meta-analysis indicated that a diet with low dietary advanced glycation end products (AGEs) had a great effect on insulin resistance, particularly in patients with diabetes. ${ }^{31}$ These findings suggest that an evidence-based plan for energy intake should be considered when designing policies for obesity management among adults.

This study has several limitations. First, this study was a single-center investigation, and the enrolled participants were primarily residing in the city. Further investigations should be conducted as multi-center studies that include various areas. Second, WC was used as an important index to reflect abdominal obesity; however, another measure, such as the visceral fat index, which may reflect the visceral adipose distribution more accurately, should be compared with WC to determine the accuracy of this measure. Finally, a cohort study is warranted to further explore the associations between different obesity patterns and the risk of developing diabetes. 


\section{Conclusion}

In summary, abdominal obesity and compound obesity are risk factors for T2DM development. More attention should be paid to preventing obesity among people younger than 60 years, and increased control of cigarette or alcohol abuse should also be considered. With the increasingly high burden of diabetes in China, obesity management should be established to prevent or delay the development of T2DM.

\section{Ethical Approval and Informed Consent}

This study adhered to the principles of the Declaration of Helsinki and was approved by the Ethics and Research Committee of Huadong Sanatorium Health Examination Center (NO.ECHS2021-03), as this study was carried out anonymously, Patient data were analysed with strict confidentiality and used only for scientific purposes. Thus, the requirement for informed consent was therefore waived.

\section{Acknowledgments}

This study received the assistance of the Health Management Centre of Huadong Sanatorium. We would also like to thank all authors and participants for their contributions.

\section{Funding}

We had no funding support for this work.

\section{Disclosure}

All authors have no conflicts of interest in this work.

\section{References}

1. Ramachandran A, Ma RC, Snehalatha C. Diabetes in Asia. Lancet. 2010;375(9712):408-418. doi:10.1016/S0140-6736(09)60937-5

2. Nanditha A, Ma RC, Ramachandran A, et al. Diabetes in Asia and the Pacific: implications for the global epidemic. Diabetes Care. 2016;39 (3):472-485. doi:10.2337/dc15-1536

3. Ma R. Epidemiology of diabetes and diabetic complications in China. Diabetologia. 2018;61(6):1249-1260. doi:10.1007/s00125-018-4557-7

4. Ma RC, Lin X, Jia W. Causes of type 2 diabetes in China. Lancet Diabetes Endocrinol. 2014;2(12):980-991. doi:10.1016/S22138587(14)70145-7

5. Ortega MA, Fraile-Martinez O, Naya I, et al. Type 2 diabetes mellitus associated with obesity (diabesity). The central role of gut microbiota and its translational applications. Nutrients. 2020;12(9):2749. doi:10.3390/nu12092749

6. Hermanides J, Cohn DM, Devries JH, et al. Venous thrombosis is associated with hyperglycemia at diagnosis: a case-control study. $J$ Thromb Haemost. 2009;7(6):945-949. doi:10.1111/j.15387836.2009.03442.x
7. Hou X, Liu Y, Lu H, et al. Ten-year changes in the prevalence of overweight, obesity and central obesity among the Chinese adults in urban Shanghai, 1998-2007 - comparison of two cross-sectional surveys. BMC Public Health. 2013;13:1064. doi:10.1186/14712458-13-1064

8. Xi B, Liang Y, He T, et al. Secular trends in the prevalence of general and abdominal obesity among Chinese adults, 1993-2009. Obes Rev. 2012;13(3):287-296. doi:10.1111/j.1467-789X.2011.00944.x

9. Du T, Sun X, Yin P, et al. Increasing trends in central obesity among Chinese adults with normal body mass index, 1993-2009. BMC Public Health. 2013;13:327. doi:10.1186/1471-2458-13-327

10. Niu J, Seo DC. Central obesity and hypertension in Chinese adults: a 12-year longitudinal examination. Prev Med. 2014;62:113-118. doi:10.1016/j.ypmed.2014.02.012

11. Twig G, Afek A, Derazne E, et al. Diabetes risk among overweight and obese metabolically healthy young adults. Diabetes Care. 2014;37(11):2989-2995. doi:10.2337/dc14-0869

12. Casanueva F, Moreno B, Rodriguez-Azeredo R, et al. Relationship of abdominal obesity with cardiovascular disease, diabetes and hyperlipidaemia in Spain. Clin Endocrinol. 2010;73(1):35-40. doi:10.1111/ j.1365-2265.2009.03727.x

13. Jia W, Weng J, Zhu D, et al. Standards of medical care for type 2 diabetes in China 2019. Diabetes Metab Res Rev. 2019;35(6):e3158. doi:10.1002/dmrr.3158

14. Chobanian AV, Bakris GL, Black HR, et al. Seventh report of the joint national committee on prevention, detection, evaluation, and treatment of high blood pressure. Hypertension. 2003;42 (6):1206-1252. doi:10.1161/01.HYP.0000107251.49515.c2

15. Chung G, Yu R, Ho S, et al. Prospective association of obesity patterns with subclinical carotid plaque development in early postmenopausal Chinese women. Obesity. 2020;28(7):1342-1350. doi:10.1002/oby.22820

16. National Clinical Research Center of Geriatric. [Expert consensus of the diagnosis and treatment measures for the Chinese elderly patients with type 2 diabetes (2018 Edition)]. Zhonghua Nei Ke Za Zhi. 2018;57(9):626-641. [Chinese]. doi:10.3760/cma.j.issn.05781426.2018.09.004

17. Alberti KG, Zimmet P, Shaw J. The metabolic syndrome--a new worldwide definition. Lancet. 2005;366(9491):1059-1062. doi:10.1016/S0140-6736(05)67402-8

18. Neeland IJ, Turer AT, Ayers CR, et al. Body fat distribution and incident cardiovascular disease in obese adults. $\mathrm{J} \mathrm{Am} \mathrm{Coll} \mathrm{Cardiol.}$ 2015;65(19):2150-2151. doi:10.1016/j.jacc.2015.01.061

19. Hayashi T, Boyko EJ, McNeely MJ, et al. Visceral adiposity, not abdominal subcutaneous fat area, is associated with an increase in future insulin resistance in Japanese Americans. Diabetes. 2008;57 (5):1269-1275. doi:10.2337/db07-1378

20. Chen ME, Chandramouli AG, Considine RV, et al. Comparison of $\beta$ cell function between overweight/obese adults and adolescents across the spectrum of glycemia. Diabetes Care. 2018;41(2):318-325. doi:10.2337/dc17-1373

21. Marzetti E, Calvani R, Tosato M, et al. Sarcopenia: an overview. Aging Clin Exp Res. 2017;29(1):11-17. doi:10.1007/s40520-016-0704-5

22. Kim JH, Lim S, Choi SH, et al. Sarcopenia: an independent predictor of mortality in community-dwelling older Korean men. J Gerontol A Biol Sci Med Sci. 2014;69(10):1244-1252. doi:10.1093/gerona/glu050

23. Jaakkola JM, Rovio SP, Pahkala K, et al. Childhood exposure to parental smoking and life-course overweight and central obesity. Ann Med. 2021;53(1):208-216. doi:10.1080/07853890.2020.1853215

24. An SY, Kim SY, Oh DJ, et al. Obesity is positively related and tobacco smoking and alcohol consumption are negatively related to an increased risk of thyroid cancer. Sci Rep. 2020;10(1):19279. doi:10.1038/s41598-020-76357-y

25. Mokdad AH, Ford ES, Bowman BA, et al. Prevalence of obesity, diabetes, and obesity-related health risk factors, 2001. JAMA. 2003;289(1):76-79. doi:10.1001/jama.289.1.76 
26. Bennet L, Stenkula K, Cushman SW, et al. BMI and waist circumference cut-offs for corresponding levels of insulin sensitivity in a Middle Eastern immigrant versus a native Swedish population-the MEDIM population based study. BMC Public Health. 2016;16 (1):1242. doi:10.1186/s12889-016-3892-1

27. Hartwig S, Kluttig A, Tiller D, et al. Anthropometric markers and their association with incident type 2 diabetes mellitus: which marker is best for prediction? Pooled analysis of four German population-based cohort studies and comparison with a nationwide cohort study. BMJ Open. 2016;6(1):e9266. doi:10.1136/bmjopen2015-009266

28. Gaman MA, Epingeac ME, Diaconu C, et al. Evaluation of oxidative stress levels in obesity and diabetes by the free oxygen radical test and free oxygen radical defence assays and correlations with anthropometric and laboratory parameters. World J Diabetes. 2020;11 (5):193-201. doi:10.4239/wjd.v11.i5.193
29. Pourrajab B, Fatahi S, Sohouli MH, et al. The effects of probiotic/ synbiotic supplementation compared to placebo on biomarkers of oxidative stress in adults: a systematic review and meta-analysis of randomized controlled trials. Crit Rev Food Sci Nutr. 2020:1-18. doi:10.1080/10408398.2020.1821166

30. Varkaneh HK, Tinsley GM, Santos HO, et al. The influence of fasting and energy-restricted diets on leptin and adiponectin levels in humans: a systematic review and meta-analysis. Clin Nutr. 2020. doi:10.1016/j.clnu.2020.10.034

31. Sohouli MH, Fatahi S, Sharifi-Zahabi E, et al. The impact of low advanced glycation end products diet on metabolic risk factors: a systematic review and meta-analysis of randomized controlled trials. Adv Nutr. 2021;12(3):766-776. doi:10.1093/advances/ nmaa 150

\section{Publish your work in this journal}

Diabetes, Metabolic Syndrome and Obesity: Targets and Therapy is an international, peer-reviewed open-access journal committed to the rapid publication of the latest laboratory and clinical findings in the fields of diabetes, metabolic syndrome and obesity research. Original research, review, case reports, hypothesis formation, expert opinion and commentaries are all considered for publication. The manuscript management system is completely online and includes a very quick and fair peer-review system, which is all easy to use. Visit http://www.dovepress.com/testimonials.php to read real quotes from published authors.

Submit your manuscript here: https://www.dovepress.com/diabetes-metabolic-syndrome-and-obesity-targets-and-therapy-journal 